\title{
LEVANTAMENTOS DA VARIAÇÃO LINGUÍSTICA EM TRÊS DOMÍNIOS DO COMPLEXO DIALETAL APURINÃ (ARÚAK)
}

\section{SURVEY OF LINGUISTIC VARIATION IN THREE DOMAINS OF THE APURINÃ DIALECTAL COMPLEX (ARAWAK)}

\author{
Bruna Fernanda Soares de Lima Padovani ${ }^{1}$ \\ Rayssa Rodrigues da Silva ${ }^{2}$ \\ Sidney da Silva Facundes ${ }^{3}$
}

\begin{abstract}
Resumo: Este trabalho tem o objetivo de apresentar um levantamento dos tipos mais representativos de variação linguística atestados na língua Apurinã (Aruák) em três domínios da língua: fonologia, morfologia e o léxico. Discutiremos os processos linguísticos envolvidos na variação, a direção das mudanças e, os fatores extralinguísticos correlatos. Mostraremos que as variações vocálicas envolvem alteamento ou síncope, que a variação consonantal envolve o apagamento da fricativa glotal e a alternância entre as consoantes líquidas, que a variação morfológica consiste, principalmente, de morfemas com sentidos semanticamente redundantes, e que os casos de variação lexical são motivados por uma rede de relações semânticas construídas a partir de sistema sociocultural e cognitivo que subjaz o fenômeno do "duplo vocabulário" Apurinã.
\end{abstract}

Palavras-chave: Variação Linguística; Apurinã; Aruák

Abctract: This work aims to present a survey of the most representative types of linguistic variation attested in the Apurinã language (Arawák) in three domains of the language: phonology, morphology and lexicon. We will discuss the linguistic processes involved in the variation, the direction of the changes and some extralinguistic correlators. We will show that the vowel variations involve vowel raising or vowel syncope, that the consonantal variation involves the glottal fricative deletion and the liquid consonants alternatios, that the morphological variation consists mainly of morphemes with semantically redundant meanings, and that the cases of lexical variation are motivated by a network of semantic relations built from a sociocultural and cognitive system that underlies the phenomenon of the "double vocabulary" in Apurinã.

Keiwords: Linguistic Variation. Apurinã. Arawak

\section{Introdução}

As considerações aqui apresentadas se relacionam ao status de três tipos de variações linguísticas (o fonológico, o morfológico e o lexical) atestados no complexo dialetal Apurinã,

\footnotetext{
${ }^{1}$ Mestre e doutoranda em Letras pela Universidade Federal do Pará (UFPA). Professora na Universidade Estadual do Pará (UEPA). E-mail: bflimapadovani@gmail.com

${ }^{2}$ E-mail: rayssa_rodrigues95@hotmail.com

${ }^{3}$ Doutor em Linguística pela State University Of New York At Buffalo. Membro de corpo editorial da Journal of Language and Linguistics e Revisor de projeto de fomento da Endangered Languages Documentation Programme. Docente da Universidade Federal do Pará (UFPA). E-mail: sfacundes@gmail.com
} 
uma das cerca de 45 línguas indígenas que permanecem vivas da família linguística Aruák (AIKHENVALD, 2005). O Apurinã é falado por um grupo de mesmo nome (se autodenomina Pupÿkary/ru) que habita tradicionalmente às margens dos tributários do rio Purus no sudoeste do Estado do Amazonas. Os membros da etnia Apurinã somam aproximadamente 7.000 pessoas (IBGE, 2010), os quais estão distribuídas em 24 terras indígenas (TI) ${ }^{4}$.

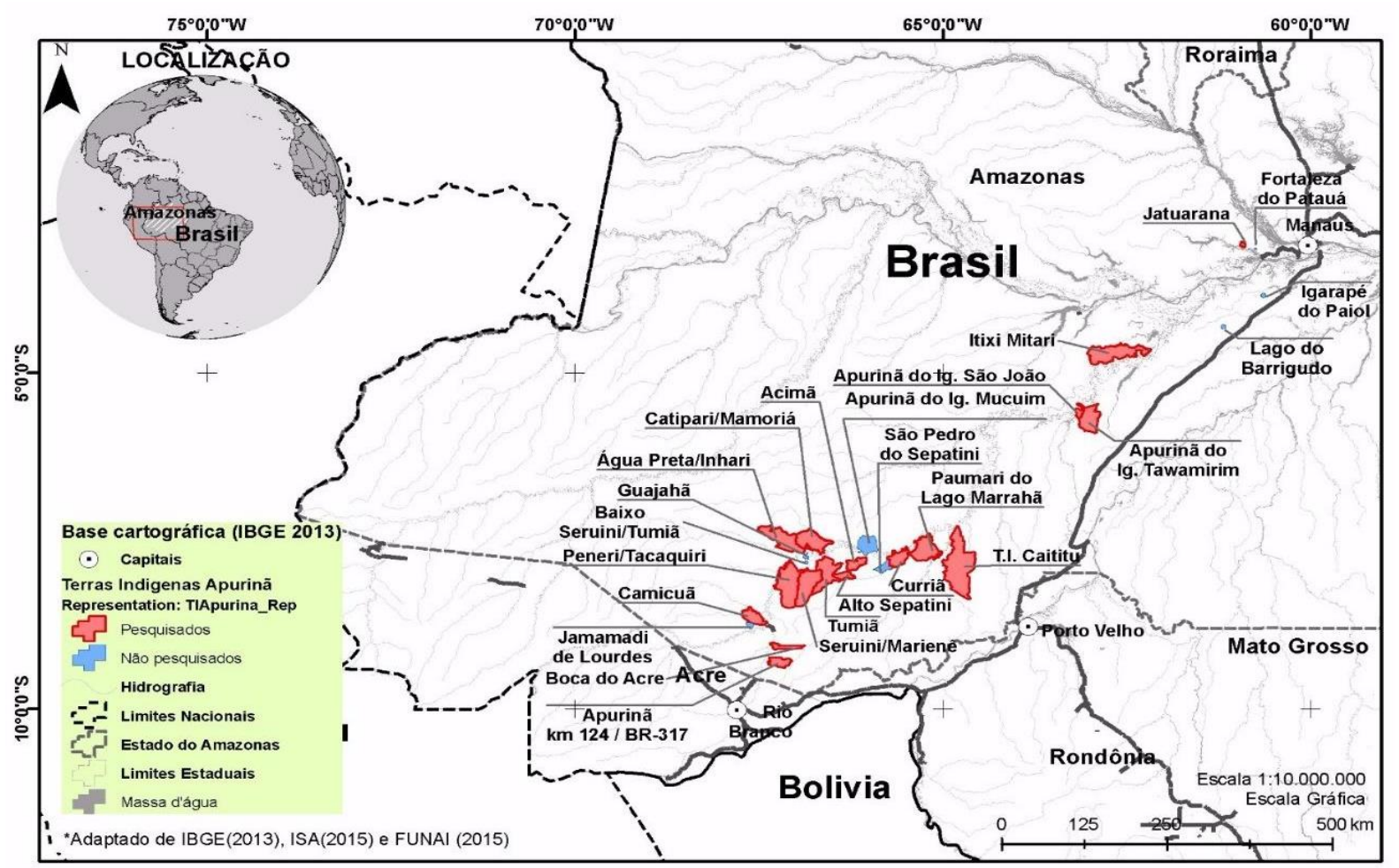

Figura 01: Mapa com a Localização Aproximada das TIs Apurinã ${ }^{5}$ (Fonte: LIMA-PADOVANI (2016, p. 30)).

Chandless (1867 apud FACUNDES, 2000) afirma que os Apurinã eram um povo inclinado à guerra, e frequentemente tinham conflitos internos, envolvendo os grupos e subgrupos de Apurinã. Esse caráter guerreiro foi apontado por Facundes (2000) como uma das principais causas da dispersão geográfica desse povo. A principal estratégia utilizada pelos indivíduos Apurinã para evitar tais conflitos, ainda hoje, é o deslocamento para comunidades distantes. Portanto, são as migrações motivadas pelos conflitos que contribuíram para a configuração presente das comunidades Apurinã. Dada a extensão dos espaços geográficos envolvidos nessas migrações, e o período de várias gerações em que elas vêm ocorrendo, a língua apresenta distintas variedades dialetais, caracterizadas por distinções na pronúncia, na

\footnotetext{
4 Informações obtidas no site da FUNAI: http://www.funai.gov.br/index.php/indios-no-brasil/terras-indigenas. Acesso em 14 maio de 2018.

${ }^{5}$ Os pontos em vermelho correspondem a localização das TIs Apurinã que fazem parte desta pesquisa.
} 
estrutura interna das palavras, e no vocabulário (BARRETO 2007; LIMA 2013 e LIMAPADOVANI 2016) como demonstraremos neste trabalho.

Os dados abordados no presente trabalho são resultados de sucessivos trabalhos de campo realizados junto aos falantes Apurinã, entre os anos de 2013 e 2018 e complementados por dados de pesquisas anteriores (FACUNDES 2000, BARRETO 2007 e BRANDÃO 2006). O estudo se articula no quadro teórico-metodológico da Sociolinguística Variacionista (LABOV, [1972] 2008), nos métodos da Linguística de Corpus (BIBER, CONRAD e REPEPEN, 1988) e da Linguística Histórica (CAMPBELL, 1999). Seguindo esses modelos, utilizamos também os conceitos e métodos tradicionalmente usados na descrição de línguas (KIBRIK 1977 e VAUX e COOPER 1999).

\section{A Variação Linguística em Apurinã}

Em Apurinã, a variação ocorre em diversos níveis da análise linguística, em que destacamos os seguintes domínios: fonológico, morfológico e a lexical. Nas subseções a seguir, descreveremos de forma breve esses tipos de variação apresentando exemplos na língua, bem como sua distribuição geográfica.

\subsection{Variação Fonológica}

A variação fonológica na língua pode ser vista no emprego de consoantes e vogais, sendo que as vogais podem variar quanto à qualidade, e nasalidade (ou posição da nasalidade). Neste trabalho, buscamos estabelecer a distribuição das variações fonológicas que se caracterizam como as variações mais distintivas nas variedades do Apurinã, além de descrever a direção das mudanças de alguns processos fonológicos envolvidos na variação.

O primeiro caso a ser considerado é a variação entre a vogal média anterior /e/ e a vogal alta central /ì/. Segundo Barreto (2007), essa variação é bastante informativa, pois é a variação fonológica mais frequente atestada na língua Apurinã. Entretanto, não pudemos determinar em que ambientes essa variação ocorre, uma vez que ela pode se realizar após diferentes consoantes, no início, meio e no final de palavras. No Quadro 01 reproduzimos alguns desses itens: 


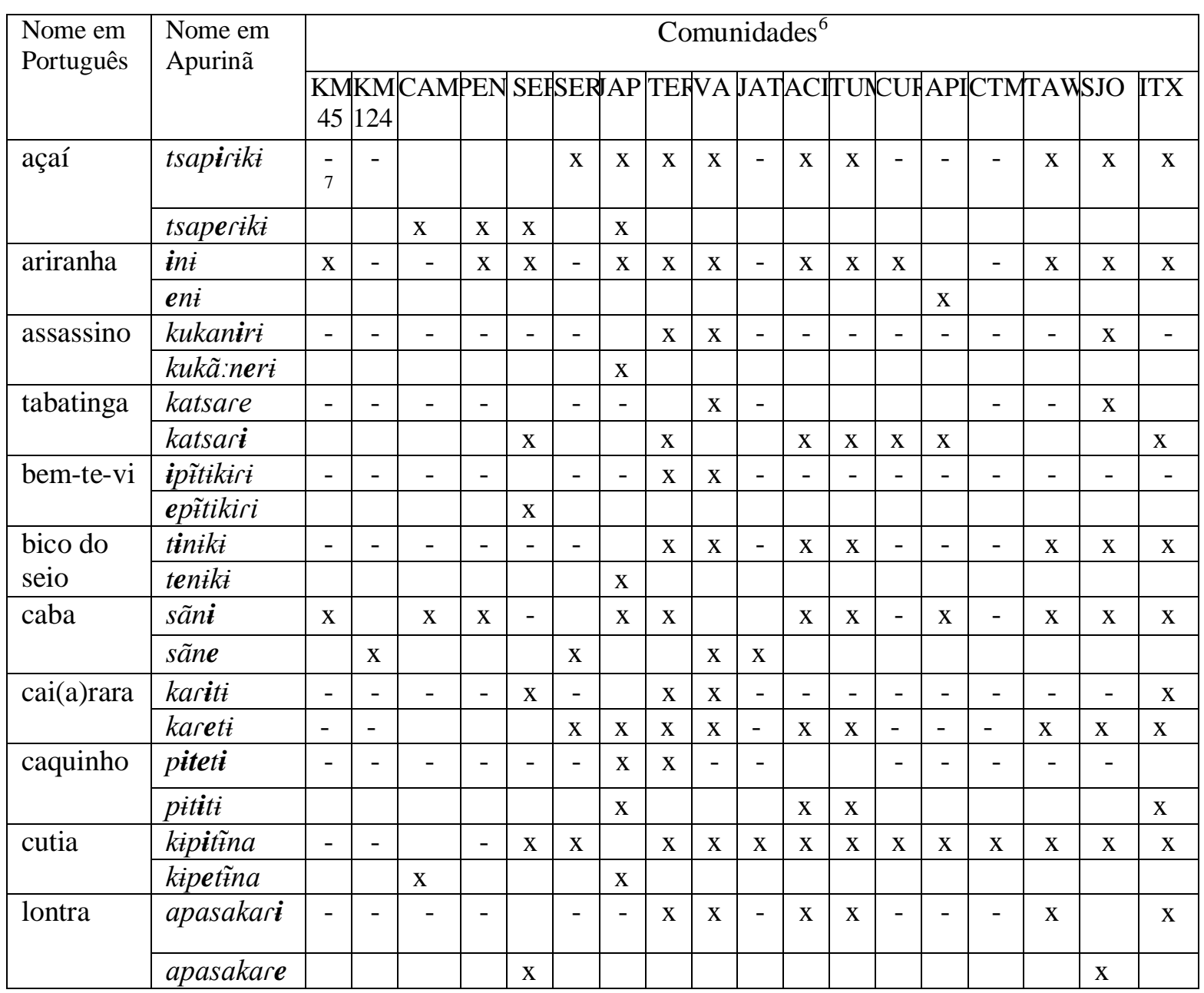

Quadro 01: Amostra parcial dos casos de variação entre /ì/ /e/ (Fonte: Adaptado de LIMA-PADOVANI (2016, p. 66)).

Em um estudo comparativo de Apurinã baseado em documentos antigos, Facundes, Neves e Lima-Padovani (2015) confirmam que a variação entre /e/ e /it/ também é a variação fonológica mais frequente na língua, e é mais ou menos unidirecional - ou seja, há muitos casos de formas com o /i/ no Apurinã atual que correspondem a /e/ nos documentos antigos, mas há também muitas formas em que o /e/ nos dados antigos não corresponde a /i/ no Apurinã atual. Provavelmente em um dado momento no passado, houve variação entre /e/ e /i//, o que posteriormente transformou-se em mudança linguística, na qual a forma /e/ estava associada a

\footnotetext{
${ }^{6}$ Abreviaturas dos nomes da comunidades Apurinã que compõem os quadros deste trabalho: ACI = Acimã; API: Água Preta/Inhari; CAM = Camicuã; CTM = Catipari/Mamoriá; CUR = Curriã; F = Feminino; JAP = Japiim; ITX = Itixi Mitari; JAT = Jatuarana; KM45 = Kilômetro 45; KM124 = Kilômetro 124; M = Masculino; PEN = Peneri; $\mathrm{SG}=$ Singular = SJO = São José; SEP = Sepatini; SER = Seruini; VA = Vista Alegre; TAW = Tawamirim; TER $=$ Terrinha; TUM = Tumiã.

${ }^{7}$ Tal símbolo (-) encontrado nos quadros onde se apresenta a distribuição das variantes pelas comunidades, indica que o conceito em questão não foi verificado ou os colaboradores não forneceram nenhuma variante na comunidade em que o símbolo aparece.
} 
determinado grupo de falantes e a forma /i/ a outro. Rodrigues (2015) também trata da variação entre /e/ e /i//, descrito como alçamento vocálico, no qual há uma elevação na altura das vogais médias altas que passam a se realizar como vogais altas.

O segundo caso a ser considerado é uma das marcas fonológicas mais importantes na distinção entre as diferentes variedades da língua Apurinã, e que consiste na presença ou ausência da fricativa glotal, /h/. Esse fonema apresenta uma distribuição fonotática restrita, pois apenas ocorre no início de algumas palavras, exceto se for precedido pela forma clítica $n u=$ '1sg' (FACUNDES, 2000), como podemos observar no Quadro abaixo:

\begin{tabular}{|c|c|c|c|c|c|c|c|c|c|c|c|c|c|c|c|c|c|c|c|c|}
\hline \multirow{3}{*}{$\begin{array}{l}\text { Nome em } \\
\text { Português }\end{array}$} & \multirow{3}{*}{$\begin{array}{l}\text { Nome em } \\
\text { Apurinã }\end{array}$} & \multicolumn{19}{|c|}{ Comunidades } \\
\hline & & KM & KM & CAM & PEN & SEP & SER & IAP & TER & $\mathrm{VA}$ & $\mid \mathrm{JA}^{\prime}$ & & $\mathrm{ACI}$ & $\mathrm{UM}$ & CUR & $\mathrm{AP}$ & CTM & TAV & $\mathrm{SJO}$ & ITX \\
\hline & & & 124 & & & & & & & & & & & & & & & & & \\
\hline \multirow[t]{2}{*}{ abano } & aputa & & - & & $\mathrm{x}$ & $\mathrm{x}$ & - & & $\mathrm{x}$ & $\mathrm{X}$ & $\mathrm{x}$ & & $\mathrm{x}$ & $\mathrm{x}$ & & & $\mathrm{x}$ & $\mathrm{x}$ & $\mathrm{x}$ & $\mathrm{x}$ \\
\hline & hãputa & $\mathrm{x}$ & & $\mathrm{x}$ & & & & $\mathrm{x}$ & & & & & & & $\mathrm{x}$ & $\mathrm{x}$ & & & & \\
\hline \multirow[t]{2}{*}{ flor } & $\tilde{t} w i$ & & & $\mathrm{x}$ & $\mathrm{x}$ & $\mathrm{x}$ & $\mathrm{x}$ & & $\mathrm{x}$ & $\mathrm{X}$ & $\mathrm{x}$ & & $\mathrm{x}$ & $\mathrm{x}$ & & & - & $\mathrm{x}$ & $\mathrm{x}$ & $\mathrm{x}$ \\
\hline & $\boldsymbol{h} \tilde{t} w \dot{t}$ & $\mathrm{x}$ & $\mathrm{x}$ & & & & & $\mathrm{x}$ & & & & & & & $\mathrm{X}$ & $\mathrm{x}$ & & & & \\
\hline \multirow{2}{*}{$\begin{array}{l}\text { filhote } \\
\text { (gente ou } \\
\text { animal) }\end{array}$} & âkiri & & - & & - & $\mathrm{x}$ & - & & $\mathrm{x}$ & $\mathrm{x}$ & - & & $\mathrm{x}$ & $\mathrm{x}$ & & $\mathrm{X}$ & - & - & $\mathrm{x}$ & $\mathrm{x}$ \\
\hline & hãkeri & $\mathrm{x}$ & & $\mathrm{x}$ & & & & $\mathrm{x}$ & & & & & & & $\mathrm{x}$ & $\mathrm{x}$ & & & & \\
\hline \multirow{2}{*}{$\begin{array}{l}\text { grande; } \\
\text { chefe dos } \\
\text { bichos }\end{array}$} & âwithe & - & & & - & $\mathrm{x}$ & - & $\mathrm{x}$ & $\mathrm{x}$ & $\mathrm{x}$ & - & & $\mathrm{x}$ & $\mathrm{x}$ & & & $\mathrm{x}$ & $\mathrm{x}$ & $\mathrm{x}$ & $\mathrm{x}$ \\
\hline & hãwithe & & $\mathrm{x}$ & $\mathrm{x}$ & & & & $\mathrm{x}$ & & & & & & & $\mathrm{x}$ & $\mathrm{x}$ & & & & \\
\hline \multirow[t]{2}{*}{ menino } & âtakuru & & - & & - & $\mathrm{x}$ & $\mathrm{x}$ & $\mathrm{x}$ & $\mathrm{x}$ & $\mathrm{x}$ & $\mathrm{x}$ & & $\mathrm{x}$ & $\mathrm{x}$ & & $\mathrm{X}$ & $\mathrm{x}$ & $\mathrm{x}$ & $\mathrm{x}$ & $\mathrm{x}$ \\
\hline & hãtakuru & $\mathrm{x}$ & & $\mathrm{x}$ & & & & $\mathrm{x}$ & & & & & & & $\mathrm{x}$ & $\mathrm{x}$ & & & & \\
\hline \multirow[t]{2}{*}{ onça } & âkiti & & & & $\mathrm{x}$ & $\mathrm{x}$ & & $\mathrm{x}$ & & $\mathrm{x}$ & & & $\mathrm{x}$ & $\mathrm{x}$ & & & $\mathrm{x}$ & $\mathrm{x}$ & $\mathrm{x}$ & $\mathrm{x}$ \\
\hline & hãkiti & $\mathrm{x}$ & $\mathrm{x}$ & $\mathrm{x}$ & & & $\mathrm{x}$ & $\mathrm{x}$ & $\mathrm{X}$ & & $\mathrm{x}$ & & & & $\mathrm{x}$ & $\mathrm{x}$ & & & & \\
\hline \multirow[t]{2}{*}{ piranha } & $\tilde{u}: m a$ & & - & & $\mathrm{x}$ & $\mathrm{x}$ & $\mathrm{x}$ & $\mathrm{x}$ & $\mathrm{x}$ & $\mathrm{x}$ & $\mathrm{x}$ & & $\mathrm{x}$ & $\mathrm{x}$ & & $\mathrm{x}$ & $\mathrm{x}$ & $\mathrm{x}$ & $\mathrm{x}$ & $\mathrm{x}$ \\
\hline & hน̃ma & $\mathrm{X}$ & & $\mathrm{x}$ & & & & $\mathrm{x}$ & & & & & & & $\mathrm{x}$ & & & & & \\
\hline \multirow[t]{2}{*}{ sangue } & erẽka & $\mathrm{x}$ & & & $\mathrm{x}$ & $\mathrm{x}$ & $\mathrm{x}$ & & & $\mathrm{x}$ & $\mathrm{x}$ & & $\mathrm{x}$ & $\mathrm{x}$ & & & $\mathrm{x}$ & $\mathrm{x}$ & $\mathrm{x}$ & $\mathrm{x}$ \\
\hline & herẽka & & $\mathrm{x}$ & $\mathrm{x}$ & & & & $\mathrm{x}$ & $\mathrm{x}$ & & & & & & $\mathrm{x}$ & $\mathrm{x}$ & & & & \\
\hline \multirow[t]{2}{*}{ abano } & ãputa & & - & & $\mathrm{x}$ & $\mathrm{x}$ & - & & $\mathrm{X}$ & $\mathrm{x}$ & $\mathrm{x}$ & & $\mathrm{x}$ & $\mathrm{x}$ & & & $\mathrm{x}$ & $\mathrm{x}$ & $\mathrm{x}$ & $\mathrm{x}$ \\
\hline & hãputa & $\mathrm{x}$ & & $\mathrm{x}$ & & & & $\mathrm{X}$ & & & & & & & $\mathrm{x}$ & $\mathrm{x}$ & & & & \\
\hline \multirow[t]{2}{*}{ flor } & $\tilde{t} w i$ & & & $\mathrm{x}$ & $\mathrm{x}$ & $\mathrm{x}$ & $\mathrm{x}$ & & $\mathrm{x}$ & $\mathrm{x}$ & $\mathrm{x}$ & & $\mathrm{x}$ & $\mathrm{x}$ & & & - & $\mathrm{x}$ & $\mathrm{x}$ & $\mathrm{x}$ \\
\hline & $\boldsymbol{h} \tilde{t} w \dot{t}$ & $\mathrm{x}$ & $\mathrm{x}$ & & & & & $\mathrm{x}$ & & & & & & & $\mathrm{x}$ & $\mathrm{x}$ & & & & \\
\hline
\end{tabular}

Quadro 02: Amostra parcial dos casos de variação entre $/ \mathrm{h} / \sim / \varnothing /$ (Fonte: Adaptado de LIMA-PADOVANI, p. 70))

Rodrigues (2015) afirma que houve um processo de supressão, que consiste na queda de um segmento no início da palavra, e que tal fato teria gerado variedades distintas na língua, algumas com a fricativa glotal /h/ e outras não. Lima-Padovani (2016), ainda, demosntra que há duas palavras iniciadas pelo segmento /h/, (h)ãty 'um' e (h)ĩthe 'vocês', que ocorrem mesmo em comunidades onde predominantemente não se faz uso da fricativa glotal. Nas comunidades: 
Itixi Mitari, Japiim, São João, Tawamirim, Terrinha e Vista Alegre o fonema /h/ está presente nas palavras hãty e hĩthe; nas comunidades São José e Peneri constam apenas a palavra hĩthe. De acordo com a autora, os documentos antigos comprovam que a fricativa glotal já existia no Apuinã antigo, inclusive num estágio que precedeu o surgimento de Apurinã, Piro e Iñapari como línguas diferentes; trata-se, portanto, da retenção parcial de um fonema na língua. Dessa perda significativa em Apurinã originou-se, segundo Facundes e Brandão (2005), a nasalidade espontânea na língua em pelo menos alguns casos. /h/ seria, então, um caso de retenção e não de inovação no Apurinã.

Além da variação fonológica, há uma situação particular de variação fonética que é também marcante na língua e que envolve o fonema /r/. Em Apurinã, esse fonema apresenta dois alofones [r] e [l]. Atestamos que em algumas variedades do Apurinã o fonema /r/ pode realizar-se como [1] quando está diante da vogal alta central /i/ ou da vogal alta anterior /i/, como podemos verificar no quadro a seguir. Apesar de fonética, tal variação constitui um importante marcador dialetal, dada a sua consistente distribuição em certas variedades da língua:

\begin{tabular}{|c|c|c|c|c|c|c|c|c|c|c|c|c|c|c|c|c|c|c|c|c|c|}
\hline \multirow{2}{*}{$\begin{array}{l}\text { Nome em } \\
\text { Português }\end{array}$} & \multirow{2}{*}{$\begin{array}{l}\text { Nome em } \\
\text { Apurinã }\end{array}$} & \multicolumn{20}{|c|}{ Comunidades } \\
\hline & & \multicolumn{4}{|c|}{ KM } & \multicolumn{3}{|c|}{ SEPSERIAP } & \multicolumn{3}{|c|}{ TERVA } & JAT & $\mathrm{ACI}$ & \multicolumn{2}{|c|}{ TUMCUR } & \multicolumn{2}{|c|}{ API } & \multicolumn{2}{|c|}{ CTNTAW } & \multicolumn{2}{|c|}{ SJO ITX } \\
\hline \multirow[t]{2}{*}{ cachorro } & anãpãnari & $\mathrm{X}$ & $\mathrm{x}$ & $\mathrm{x}$ & & & - & $\mathrm{x}$ & $\mathrm{X}$ & - & & $\mathrm{X}$ & $\mathrm{X}$ & $\mathrm{X}$ & $\mathrm{x}$ & & $\mathrm{x}$ & $\mathrm{X}$ & $\mathrm{x}$ & $\mathrm{x}$ & $\mathrm{X}$ \\
\hline & anãpãnali & & & & $\mathrm{X}$ & $\mathrm{x}$ & & & & & & & & & & & & & & & \\
\hline \multirow[t]{2}{*}{ cigarra } & kĩturi & $\mathrm{X}$ & - & $\mathrm{x}$ & & & & $\mathrm{x}$ & $\mathrm{X}$ & $x$ & & & $\mathrm{X}$ & $\mathrm{X}$ & $\mathrm{X}$ & 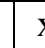 & $\mathrm{x}$ & $\mathrm{X}$ & $\mathrm{x}$ & $\mathrm{x}$ & $\mathrm{X}$ \\
\hline & kituli & & & & X & $\mathrm{X}$ & $\mathrm{X}$ & & & & & $X$ & & & & & & & & & \\
\hline \multirow{2}{*}{$\begin{array}{l}\text { flexa } \\
\text { pronta }\end{array}$} & katsũta:lit & - & - & - & - & $\mathrm{x}$ & - & & & & & - & & - & - & . & - & & & & \\
\hline & katsĩta:ri & & & & & & & $\mathrm{X}$ & $\mathrm{X}$ & $X$ & & & $\mathrm{X}$ & & & & & $\mathrm{X}$ & $\mathrm{X}$ & $\mathrm{X}$ & $\mathrm{X}$ \\
\hline \multirow[t]{2}{*}{ igarapé } & kawãari & - & - & - & & & - & - & $\mathrm{X}$ & $x$ & & - & $\mathrm{X}$ & $\mathrm{x}$ & - & - & - & - & $\mathrm{X}$ & $\mathrm{x}$ & $\mathrm{X}$ \\
\hline & kawãali & & & & $\mathrm{X}$ & $\mathrm{X}$ & & & & & & & & & & & & & & & \\
\hline \multirow[t]{2}{*}{ irara } & mapaãnari & $\mathrm{X}$ & - & $\mathrm{x}$ & & & - & $\mathrm{x}$ & $\mathrm{x}$ & $x$ & & - & $\mathrm{x}$ & $\mathrm{X}$ & - & 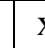 & $\mathrm{x}$ & $\mathrm{X}$ & $\mathrm{x}$ & $\mathrm{x}$ & $\mathrm{X}$ \\
\hline & mapaãnali & & & & $\mathrm{x}$ & $\mathrm{x}$ & & & & & & & & & & & & & & & \\
\hline \multirow[t]{2}{*}{ jenipapo } & ymamari & $\mathrm{X}$ & - & $\mathrm{x}$ & & & - & $\mathrm{X}$ & $\mathrm{X}$ & $x$ & & $\mathrm{x}$ & $\mathrm{X}$ & $\mathrm{X}$ & $\mathrm{x}$ & 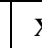 & $\mathrm{x}$ & $\mathrm{X}$ & $\mathrm{x}$ & $\mathrm{x}$ & $\mathrm{X}$ \\
\hline & ymamali & & & & $\mathrm{X}$ & $\mathrm{X}$ & & & & & & & & & & & & & & & \\
\hline \multirow[t]{2}{*}{ preto } & pumamari & $\mathrm{X}$ & $\mathrm{X}$ & $\mathrm{x}$ & & & & $\mathrm{x}$ & $\mathrm{X}$ & $x$ & & $\mathrm{x}$ & $\mathrm{X}$ & $\mathrm{X}$ & $\mathrm{x}$ & 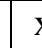 & $\mathrm{x}$ & $\mathrm{X}$ & $\mathrm{x}$ & $\mathrm{X}$ & $\mathrm{X}$ \\
\hline & pumamali & & & & $\mathrm{X}$ & $\mathrm{X}$ & $\mathrm{X}$ & & & & & & & & & & & & & & \\
\hline \multirow[t]{2}{*}{ queixada } & irari & $\mathrm{X}$ & $\mathrm{X}$ & $\mathrm{x}$ & & & & $\mathrm{X}$ & $\mathrm{x}$ & $x$ & & $\mathrm{X}$ & $\mathrm{X}$ & $\mathrm{X}$ & $\mathrm{x}$ & & $\mathrm{x}$ & $\mathrm{X}$ & $\mathrm{x}$ & $\mathrm{X}$ & $\mathrm{X}$ \\
\hline & iralit & & & & $\mathrm{X}$ & $\mathrm{X}$ & $\mathrm{X}$ & & & & & & & & & & & & & & \\
\hline \multirow[t]{2}{*}{ cachorro } & anãpãnari & $\mathrm{X}$ & $\mathrm{X}$ & $\mathrm{x}$ & & & - & $\mathrm{X}$ & $\mathrm{X}$ & - & & $\mathrm{X}$ & $\mathrm{X}$ & $\mathrm{x}$ & $\mathrm{X}$ & $x$ & $\mathrm{x}$ & $\mathrm{X}$ & $\mathrm{X}$ & $\mathrm{X}$ & $\mathrm{X}$ \\
\hline & anãpãnali & & & & $\mathrm{X}$ & $\mathrm{X}$ & & & & & & & & & & & & & & & \\
\hline
\end{tabular}

Quadro 3: Amostra parcial variação entre [r] e [I] (Fonte: LIMA-PADOVANI (2016, p. 73)). 
Há evidências históricas de que o processo em questão atuou em outro momento, tendo sido atestado nos dados de Koch-Grünberg (1919). Diante disso, podemos levantar como hipótese que essa variação é antiga na língua e que hoje se preserva nas variedades possivelmente mais arcaicas.

Nos casos de variação fonológica subsequentes, apresentaremos casos de variação que não seguem um padrão extritamente fonológico, pois tais casos de variação restringem-se a algumas palavras, sendo portanto, lexicalmente condicionados. Importante ressaltar que tais varições são marcas distintivas dos diferentes dialetos da língua Apurinã.

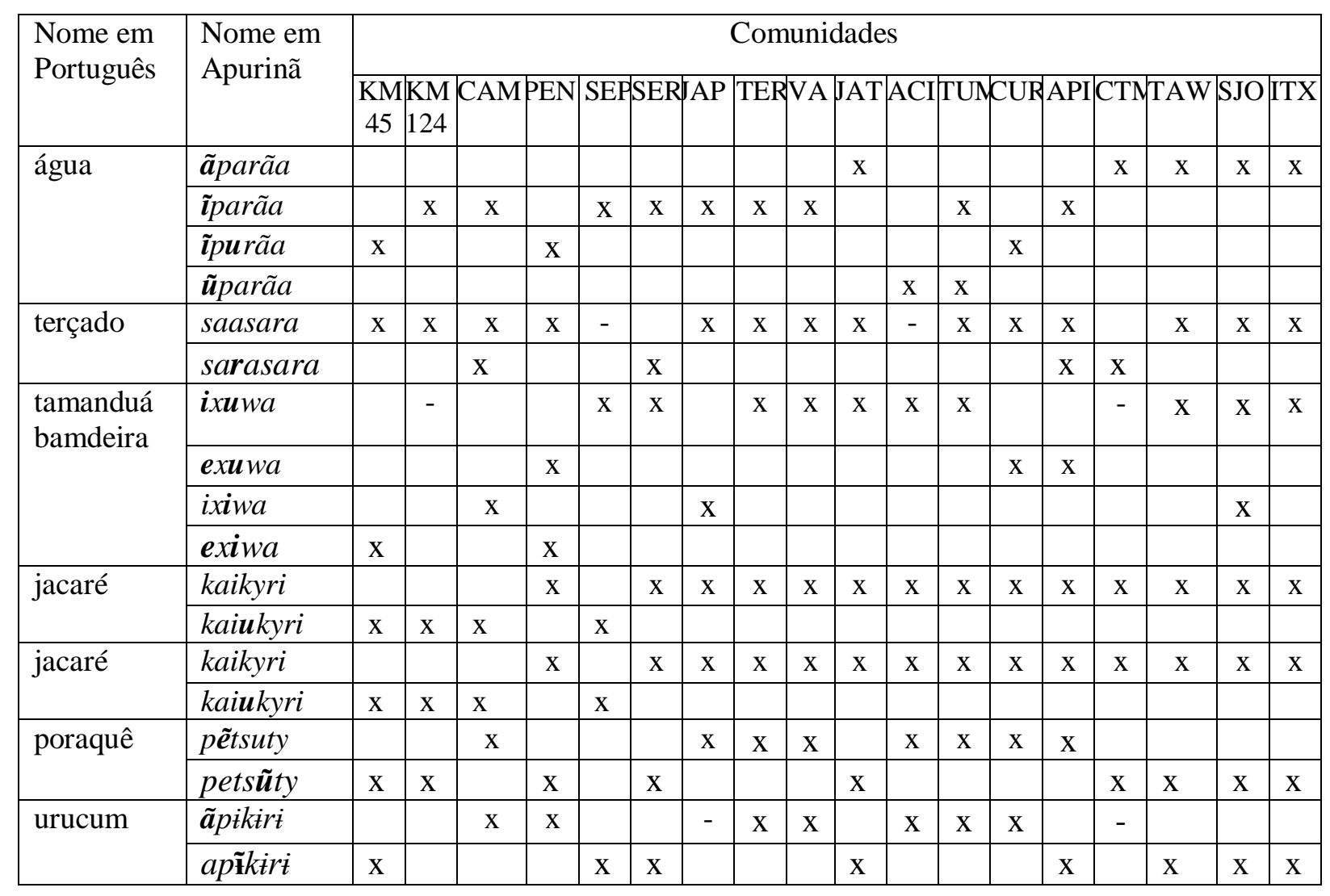

Quadro 04: Casos de variação fonológica lexicalmente condicionados (Fonte: LIMA-PADOVANI (2016, p. 76))

O primeiro item desse quadro é a palavra para 'água'. Há quatro formas distintas facilmente reconhecíveis pelos falantes da língua: /ãparãa/, /ĩparãa/, /ĩpurãa/, e /ũparãa/, cada uma dessas variantes está associada a comunidades distintas. Um dado importante em relação a esta variante é o fato de termos identificado uma forma mais semelhante à segunda forma em Polak: Imbarán, e duas formas mais semelhantes à terceira, em Koch-Grünberg: impurãna, e em Nimuendajú: įborã (FACUNDES, NEVES \& LIMA-PADOVANI, 2015), o que sugere essa segunda forma ser a mais antiga na língua. Rodrigues (2015) trata essa variação como fonologicamente pelo processo de alçamento ou posteriorização. Teriamos, portanto, a ocorrência das seguintes direções de mudança: $\mathrm{a} \square \mathrm{u}, / \tilde{\mathbf{1}} \square \mathrm{u}$, lexicalmente condicionadas. 
No segundo item do Quadro verificamos duas formas para 'terçado': /sarasara/ e /saasara/. A variação ocorre neste item em virtude da supressão (síncope) do fonema /r/ na segunda forma. Vale ressaltar que, ao examinarmos os documentos antigos, identificamos em Koch-Grünberg a forma sarasara. O que indica que esta forma é a mais antiga em Apurinã.

No terceiro item identificamos quatro formas para designar 'tamanduá bandeira': /ixu $w a /$, lexuwal, /ixiwa/ e /exiwal. Essas variantes apresentam uma alternância entre as vogais /i/ e /e/ no início palavra e entre /u/ e /i/ no meio da palavra. Identificamos, ainda, nos documentos antigos duas formas semelhantes à terceira forma, em Koch-Grünberg: išiua e em, Nimuendajú: isyú:a. O que sugere que a forma /ixiwal é a mais antiga.

O quarto item é a forma que designa 'mutum': /irãkal e /irẽka/. Observa-se que a

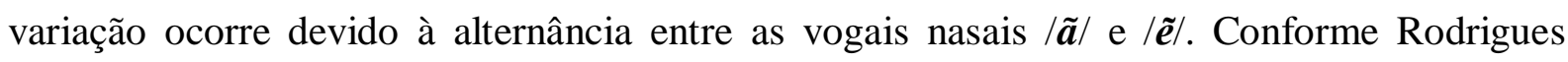
(2015), trata-se de mais um exemplo de alçamento vocálico, em que a vogal nasal aberta /ãa varia para a vogal nasal meio-fechada /ẽ/. A análise como alçamento e não abaixamento motivase pelos dados atestados em fontes antigas, onde identificamos duas formas que se aproximam da primeira variedade variante, são elas: Koch-Grünberg: iraňká, Nimuendajú: iran்ga. O que indica que /irãkal é a forma mais antiga.

Para o conceito 'jacaré' atestamos duas formas: /kaikyry/ e /kaiukyry/. Nota-se que a variação envolve a presença/ausência da vogal alta posterior /u/. Houve o apagamento (síncope) da vogal/u/ (RODRIGUES, 2015). As formas atestadas nos documentos antigos são káikiri em Nimuendajú, kàyókere em Koch-Grünberg, e Cayukŷrŷ em Polak. Observa-se que a forma identificada em Nimuendajú se assemelha à primeira forma e às formas atestadas em KochGrünberg e Polak se assemelham a segunda forma. Identificamos também cognatos em Piro e Iñapari, são eles: kajukiri e kfijoçru respectivamente. Nota-se que esses cognatos se assemelham à forma /kaiukyry/. O fato dessa forma ter sido identificada nos documentos antigos e apresentar cognatos em outras línguas da família Aruák indica ser ela a mais antiga.

O sétimo e o oitavo item do Quadro variam em termos da posição da vogal nasal. A forma para 'poraquê', pode ser /pẽtsuty/e/petsũty/. O mesmo ocorre com a palavra que designa

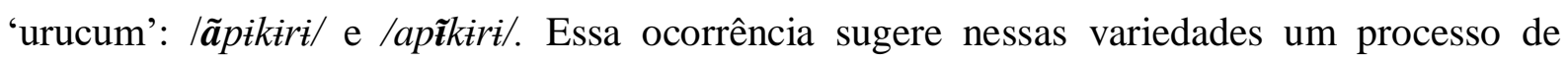
metátese prosódica, que consiste na inversão da posição da nasalidade vocálica. É possível que esse processo tenha sido comum na língua no passado, mas que tenha desaparecido e deixado diferentes variantes em comunidades distintas, algumas com a vogal nasal em uma posição, outras com a vogal nasal em outra posição (LIMA-PADOVANI, 2016, p. 78-79). 
A partir da ótica variacionista, observamos nos casos de variação fonológica aqui citados que os fonemas podem ter diferentes realizações fonéticas ou monofofonêmicas que se alternam no mesmo contexto linguístico. A escolha entre as formas que alternam entre si, as variantes, se dá a partir de influências internas e/ou externas à língua. Em Apurinã, verificamos que a distribuição geográfica das comunidades afeta diretamente o processo de variação fonológica na língua, além de outros fatores tais como faixa etária.

Nesta seção, vimos os casos mais robustos de variação fonológica em Apurinã, bem como seus respectivos processos fonológicos. Os Quadros acima possibilitaram a visualização de como as variações estão distribuídas entre as comunidades mencionadas e facilitou a descrição dos processos fonológicos envolvendo a direção das mudanças. A seguir iremos apresentar os casos de variação morfológica nessa língua.

\subsection{Variação morfológica}

Os casos de variação morfológica em Apurinã envolvem, na sua maior parte, o uso de nomes classificatórios ${ }^{8}$, e, em alguns casos, marcas de gênero (BARRETO, 2007). Em pesquisa recente, Lima-Padovani (2016) identificou também casos de variação morfológica envolvendo o paradigma pronominal de marcação de sujeito e a redução da estrutura de algumas palavras.

A variação envolvendo nomes classificatórios se deve à sua função parcialmente redundante, do ponto de vista semântico, já que as informações agregadas a esses nomes podem ser geralmente inferidas a partir do contexto semântico ou pragmático. Por exemplo, na palavra txuwiriane-ke 'gato maracajá', a sílaba final -ke é o nome classificatório para elementos de forma fina, alongada e, em geral, flexível. Nessa palavra, -ke descreve propriedades físicas do gato maracajá, semanticamente desnecessárias à identificação do animal (ao menos em contextos gerais em que essas propriedades não sejam fundamentais à identificação do referente) e, portanto, redundantes; desse modo, a não inclusão desse nome classificatório na nomeação desse animal não prejudica a sua identificação referencial. Por isso, é de se esperar que alguns falantes façam uso dessa redundância e outros não. A seguir, apresentamos exemplos da variação morfológica envolvendo nomes classificatórios:

\footnotetext{
${ }^{8}$ De acordo com Facundes (2000), embora os nomes classificatórios assemelham-se, em alguns aspectos, aos classificadores nominais, o termo "nomes classificatórios" é usado porque esses morfemas se assemelham mais aos "class terms" que, segundo DeLANCEY (1986, apud FACUNDES, 2000, p. 187), dão origem a classificadores numerais em línguas do sudeste asiático.
} 


\begin{tabular}{|c|c|c|}
\hline $\begin{array}{l}\text { Nome em } \\
\text { português }\end{array}$ & Formas Apurinã & Diferença Formal \\
\hline \multirow[t]{2}{*}{ cachorro } & anãpa & \\
\hline & anãpanary & -na 'faz referência a formas cilindricas e finas' \\
\hline \multirow[t]{2}{*}{ castanha } & maky & - \\
\hline & makyta & -ta'referência a borda arredondada' \\
\hline \multirow[t]{2}{*}{ cipó ambé } & xĩpi & - \\
\hline & xĩpitsa & $\begin{array}{l}\text {-tsa' forma fina, alongada e entrelaçada, em geral, é usado em } \\
\text { nome para cipó' }\end{array}$ \\
\hline \multirow[t]{2}{*}{ sapo } & kura & - \\
\hline & kuraty & -ty 'formas grandes' \\
\hline \multirow[t]{2}{*}{ gato maracajá } & txuwiriẽne & - \\
\hline & txuwiriẽneke & $-k e^{`}$ forma fino, alongado e flexível' \\
\hline \multirow[t]{2}{*}{ piranha } & (h) $\tilde{u} m a$ & - \\
\hline & (h) ümaky & -ky 'forma esférica; pequena' \\
\hline \multirow[t]{2}{*}{ rato pequeno } & kÿyry & - \\
\hline & kÿyryky & -ky 'pequeno e arrendondado' \\
\hline
\end{tabular}

Quadro 05: Amostra parcial decasos de variação morfológica envolvendo nomes classificatórios (Fonte: Adaptado de LIMA-PADOVANI, (2016, p. 80)).

Verificamos esses dados com diferentes falantes e em diferentes comunidades e observamos que não há obrigatoriedade gramatical de uso dos termos classificatórios nas comunidades em que ocorrem.

No que corresponde às marcas pronominais de gênero, estas são mais uma das propriedades morfológicas em Apurinã que distinguem os nomes das demais partes do discurso (classe de palavras) na língua. Segundo Facundes (2000, p. 219) a maioria dos nomes em Apurinã não é marcada morfologicamente para o gênero. Podemos supor, portanto, que o fato de que o gênero de certos nomes pode ser identificado a partir do contexto semântico ou pragmático-discursivo permite que tais nomes não sejam obrigatoriamente marcados morfologicamente para o gênero. A marca só ocorre com nomes em que o gênero está relacionado ao sexo biológico o que, por sua vez, gera a variação morfológica de algumas palavras. Quando trazem a marca pronominal de gênero, utilizam as formas $-\boldsymbol{r y}$, que designa 'masculino', ou -ru, para o ‘feminino'. A seguir, apresentaremos alguns exemplos de variação morfológica que envolvem a marca de gênero: 
Exemplo 1: a) cachorro (anãpa anãpanary anãpanaru)

b) pica-pau $(k \tilde{u} k a \sim k \tilde{k} k a r y \sim k \tilde{u} k a r u)$

c) jacu (tũty $\sim$ tũtykyry $\sim$ tũtykyru $)$

Os pronomes em Apurinã formam a única classe de palavras cujos membros se combinam para codificar pessoa, número e gênero, ou uma combinação desses significados, dependendo do pronome em questão (FACUNDES, 2000, p. 345). Os pronomes não têm uma morfologia inerente, portanto, podem ser diferenciados morfologicamente de nomes e verbos, uma vez que dispensam a morfologia que é inerentemente nominal e/ou verbal. Os únicos formativos presos que se ligam a bases pronominais são aqueles que Facundes (2000, p. 348) agrupou na classe dos formativos presos especiais ou morfemas flutuantes. Em relação aos pronomes independentes, Facundes (2000) identificou sete morfemas, quatro no singular e três no plural, sendo que apenas a terceira pessoa do singular apresenta distinção de gênero, conforme quadro abaixo:

\begin{tabular}{|c|c|c|}
\hline \multirow{2}{*}{$\begin{array}{l}\text { Pessoa e } \\
\text { Gênero }\end{array}$} & \multicolumn{2}{|c|}{ Formas Pronominais } \\
\hline & Singular & Plural \\
\hline 1 & nuta & atha \\
\hline 2 & pithe & hỹthe \\
\hline $3 \mathrm{M}$ & ywa & \multirow[t]{2}{*}{ ynawa } \\
\hline $3 \mathrm{~F}$ & uwa & \\
\hline
\end{tabular}

Quadro 06: Pronomes independentes em Apurinã (Fonte: FACUNDES (2000, p. 346, tradução e adaptação nossa)

Os pronomes de terceira pessoal singular podem variar morfologicamente. Indentificamos a ocorrência de rywa para terceira pessoa masculino e ruwa para terceira pessoa feminino. Tais variantes parecem ocorrer apenas na comunidade do $\mathrm{Km} 45$, já que até o momento não atestamos tal forma em outras comunidades. Vejamos alguns exemplos:

\begin{tabular}{|c|c|c|}
\hline \multirow[t]{3}{*}{ Exemplo 2: } & $\begin{array}{l}\text { a) rywa } \\
\text { 3SG.M } \\
\text { Ele dormiu }\end{array}$ & $\begin{array}{l}\text { ymapeka } \\
\text { dormi }\end{array}$ \\
\hline & $\begin{array}{l}\text { b) ruwa } \\
\text { 3SG.F } \\
\text { Ela dançou }\end{array}$ & $\begin{array}{r}\text { pareka } \\
\text { dançar }\end{array}$ \\
\hline & $\begin{array}{l}\text { c) rywa } \\
\text { 3SG.M }\end{array}$ & $\begin{array}{l}\text { aupẽka } \\
\text { chegar }\end{array}$ \\
\hline
\end{tabular}


Ele chegou ontem

$\begin{array}{lll}\text { d) ruwa } & \text { aupẽka } & \text { kita } \\ \text { 3SG.F } & \text { chegar } & \text { ontem }\end{array}$

Ela chegou ontem

Além da forma ynawa, que corresponde à terceira pessoal do plural, identificamos também a ocorrência das formas ynuwa, nynawa e nynuwa. Nota-se que as formas nynawa e nynuwa são variantes morfológicas das formas ynawa e ynuwa respectivamente. Tais formas não co-ocorrem em uma mesma variedade da língua Apurinã. Quando em uma determinada comunidade se usa uma dessas quatro formas, as outras não são usadas. É importante frisar que essas formas podem ser empregadas tanto para o gênero feminino quanto masculino.

Dessa forma, para o pronome de terceira pessoa singular e plural nos temos o seguinte paradigma:

\begin{tabular}{|c|c|c|}
\hline \multirow{2}{*}{$\begin{array}{l}\text { Pessoa e } \\
\text { Gênero }\end{array}$} & \multicolumn{2}{|c|}{ Formas Pronominais } \\
\cline { 2 - 2 } & Singular & Plural \\
\hline $3 \mathrm{M}$ & $y w a$ e rywa & $\begin{array}{c}\text { ynawa, ynuwa, } \\
\text { nynawa e nynuwa }\end{array}$ \\
\hline $3 \mathrm{~F}$ & uwa e ruwa & \\
\hline
\end{tabular}

Quadro 07: Variação morfológica do paradigama de terceira pessoa singular (Fonte: LIMA-PADOVANI (2016, p. 82)).

Como mencionado no início desta seção, verificamos em pesquisa recente que a variação morfológica ocorre também com a redução da estrutura interna de algumas palavras. Alguns falantes subtraem morfemas da estrutura interna de determinadas palavras, como podemos observar nos exemplos abaixo:

Exemplo 3: a) dor (katsyywyry katsyy tsywy tsyy)

b) macaco de cheiro (amãtxuary txuary)

c) mucura (xapakury xapary)

d) sanguessuga (etyperu ety)

\subsection{Variação Lexical}

A variação lexical em Apurinã se manifesta na forma de dois parâmetros básicos da sociolinguística que estão interligados: (i) a distribuição geográfica, que é representada por 
aquelas formas que têm suas variantes linguísticas distribuídas em espaços geográficos diferentes; e (ii) a variação associada a questões ligadas à idade do falante.

Iniciemos pelo fator geográfico. Como mencionamos anteriormente, as comunidades Apurinã estão distribuídas ao longo dos tributários do rio Purus, apresentando uma larga extensão geográfica e com grandes distâncias entre si. Esse fato favorece a variação lexical na língua, fazendo com que certas variantes sejam usadas em algumas comunidades, mas não em outras. O espaço geográfico evidencia a particularidade de cada comunidade, exibindo a variedade que a língua assume de uma região para outra, como forma de caracterizar a diversidade cultural, a natureza da formação demográfica da região, e a interferência de outras línguas que se tenha feito presente naquele espaço no curso de sua história.

Os grupos de variantes analisados no Quadro 05 estão entre os principais itens lexicais que distinguem entre as diversas variedades atuais da língua Apurinã. A análise, portanto, não é exaustiva em relação ao total de variantes lexicais existentes no Apurinã atual. Analisamos apenas os parâmetros de variação geográfica que são mais informativos, isto é, aqueles que claramente funcionam como marcadores das diferentes variedades da língua:

\begin{tabular}{|c|c|c|c|c|c|c|c|c|c|c|c|c|c|c|c|c|c|c|c|c|}
\hline \multirow{2}{*}{$\begin{array}{l}\text { Nome em } \\
\text { português }\end{array}$} & \multirow{2}{*}{$\begin{array}{l}\text { Nome em } \\
\text { Apurinã }\end{array}$} & \multicolumn{19}{|c|}{ Comunidades } \\
\hline & & KM & KM & CAM & PEN & SEP & SER & $\mathrm{JAI}$ & TE & $\mathrm{V}$ & & $\mathrm{AT}$ & UM & $\mathrm{ACI}$ & CUR & API & FTM & SJO & TAW & ITX \\
\hline \multirow[t]{3}{*}{ café } & kỹpatykỹa & & & & & & & $\mathrm{x}$ & & $\mathrm{x}$ & & & $\mathrm{x}$ & & & & & & & \\
\hline & kapẽe & $\mathrm{x}$ & $\mathrm{x}$ & & $\mathrm{x}$ & $\mathrm{x}$ & $\mathrm{x}$ & $\mathrm{X}$ & $\mathrm{x}$ & $x$ & & & & $\mathrm{x}$ & $\mathrm{x}$ & $\mathrm{x}$ & & $\mathrm{x}$ & $\mathrm{x}$ & $\mathrm{x}$ \\
\hline & pumamãru & & & & & & & & & & & & & & & & & $\mathrm{x}$ & $\mathrm{x}$ & $\mathrm{x}$ \\
\hline \multirow[t]{2}{*}{ cavalo } & ixiwãwita & $\mathrm{x}$ & & & & & $\mathrm{x}$ & $\mathrm{x}$ & & $\mathrm{x}$ & & & & $\mathrm{x}$ & & & & & & \\
\hline & kawaru & & & & & $\mathrm{x}$ & $\mathrm{x}$ & $\mathrm{x}$ & $\mathrm{x}$ & . & & & $\mathrm{x}$ & & & & & & & \\
\hline \multirow[t]{2}{*}{ farinha } & (k)atarukyry & $\mathrm{x}$ & & & & & & $\mathrm{x}$ & & $\mathrm{x}$ & & & $\mathrm{x}$ & & $\mathrm{x}$ & $\mathrm{x}$ & & $\mathrm{X}$ & $\mathrm{x}$ & $\mathrm{x}$ \\
\hline & parĩia & & & & & $\mathrm{x}$ & & & $\mathrm{x}$ & & & & & $\mathrm{x}$ & & & & & & \\
\hline \multirow{3}{*}{$\begin{array}{l}\text { gato } \\
\text { maracajá }\end{array}$} & txuwĩriãrike & & & $\mathrm{x}$ & $\mathrm{x}$ & $\mathrm{x}$ & $\mathrm{x}$ & $\mathrm{x}$ & $\mathrm{x}$ & & & $\mathrm{x}$ & $\mathrm{x}$ & & & & $\mathrm{x}$ & $\mathrm{x}$ & $\mathrm{x}$ & $\mathrm{x}$ \\
\hline & putxukari & $\mathrm{x}$ & & & & & & & & & & & & & & & & & & \\
\hline & âkiti & & & & & & & & & $\mathrm{x}$ & & & & $\mathrm{x}$ & & $\mathrm{x}$ & & & & \\
\hline \multirow[t]{2}{*}{ jacu } & $t \tilde{u} t i$ & & & & & & & $\mathrm{x}$ & $\mathrm{x}$ & $x$ & & $\mathrm{x}$ & $\mathrm{x}$ & & $\mathrm{x}$ & $\mathrm{x}$ & $\mathrm{x}$ & $\mathrm{x}$ & $\mathrm{x}$ & $\mathrm{x}$ \\
\hline & thãpukyry & $\mathrm{x}$ & $\mathrm{x}$ & $\mathrm{X}$ & & $\mathrm{x}$ & $\mathrm{x}$ & & & & & & & $\mathrm{X}$ & & & & & & \\
\hline \multirow[t]{2}{*}{ japó } & iũpiri & & & $\mathrm{x}$ & $\mathrm{x}$ & & $\mathrm{x}$ & $\mathrm{x}$ & $\mathrm{x}$ & $x$ & & & $\mathrm{x}$ & & $\mathrm{x}$ & $\mathrm{x}$ & $\mathrm{x}$ & & & \\
\hline & pukuru(ru) & $\mathrm{x}$ & & & & $\mathrm{x}$ & & & & & & & & $\mathrm{x}$ & & & & $\mathrm{x}$ & $\mathrm{x}$ & $\mathrm{x}$ \\
\hline
\end{tabular}




\begin{tabular}{|c|c|c|c|c|c|c|c|c|c|c|c|c|c|c|c|c|c|c|c|}
\hline \multirow[t]{2}{*}{ onça preta } & $\begin{array}{l}\text { (h)ãkiti } \\
\text { mapiãnyry }\end{array}$ & $\mathrm{x}$ & & & & & $\mathrm{x}$ & $\mathrm{x}$ & $\mathrm{x}$ & & & & $\mathrm{x}$ & & & & & & \\
\hline & $\begin{array}{l}\text { (h)ãkiti } \\
\text { pumamary }\end{array}$ & & $\mathrm{X}$ & $\mathrm{x}$ & $\mathrm{x}$ & $\mathrm{x}$ & $\mathrm{x}$ & & & $\mathrm{x}$ & $\mathrm{x}$ & $\mathrm{x}$ & & $\mathrm{x}$ & $\mathrm{x}$ & $\mathrm{X}$ & $\mathrm{x}$ & $\mathrm{X}$ & $\mathrm{X}$ \\
\hline \multirow[t]{2}{*}{$\begin{array}{l}\text { onça } \\
\text { vermelha }\end{array}$} & $\begin{array}{l}\text { (h)ãkiti } \\
\text { putsukare }\end{array}$ & $\mathrm{x}$ & $\mathrm{x}$ & $\mathrm{X}$ & & $\mathrm{x}$ & & & & & & & $\mathrm{x}$ & & & & & & \\
\hline & $\begin{array}{l}\text { (h)ãkiti } \\
\text { pũkamarary }\end{array}$ & & & & $\mathrm{x}$ & & & $\mathrm{x}$ & $\mathrm{x}$ & $\mathrm{x}$ & $\mathrm{x}$ & $\mathrm{x}$ & & $\mathrm{x}$ & $\mathrm{x}$ & $\mathrm{x}$ & $\mathrm{x}$ & $\mathrm{x}$ & $\mathrm{x}$ \\
\hline \multirow{2}{*}{$\begin{array}{l}\text { pé de } \\
\text { tucumã }\end{array}$} & xũkuruna & & & & & $\mathrm{x}$ & & & & & $\mathrm{x}$ & & & $\mathrm{x}$ & & $\mathrm{x}$ & $\mathrm{x}$ & & \\
\hline & ikawaryna & $\mathrm{x}$ & $\mathrm{X}$ & $\mathrm{x}$ & $\mathrm{x}$ & & $\mathrm{X}$ & & $\mathrm{x}$ & $\mathrm{x}$ & & $\mathrm{x}$ & $\mathrm{x}$ & & $\mathrm{x}$ & & $\mathrm{x}$ & $\mathrm{X}$ & $\mathrm{x}$ \\
\hline \multirow{2}{*}{$\begin{array}{l}\text { porco } \\
\text { doméstico }\end{array}$} & iraryãwitha & & & & & & $\mathrm{x}$ & $\mathrm{x}$ & $\mathrm{x}$ & $\mathrm{x}$ & & $\mathrm{x}$ & & & & & $\mathrm{x}$ & $\mathrm{x}$ & $\mathrm{X}$ \\
\hline & miritiãwitha & $\mathrm{x}$ & $\mathrm{x}$ & $\mathrm{x}$ & $\mathrm{x}$ & $\mathrm{x}$ & & & & & $\mathrm{x}$ & & $\mathrm{x}$ & & & & & & \\
\hline \multirow[t]{3}{*}{$\begin{array}{l}\text { quatipuru } \\
\text { roxinho }\end{array}$} & $\begin{array}{l}\text { akkiti } \\
\text { tikakiery }\end{array}$ & & & & & $\mathrm{x}$ & & & $\mathrm{x}$ & $\mathrm{x}$ & & $\mathrm{x}$ & $\mathrm{x}$ & & $\mathrm{x}$ & & $\mathrm{x}$ & $\mathrm{X}$ & $\mathrm{X}$ \\
\hline & $\begin{array}{l}\text { iübitiri } \\
\text { pumamakyry }\end{array}$ & & & & & & & & & & & $\mathrm{X}$ & & & & & & $\mathrm{X}$ & $\mathrm{X}$ \\
\hline & kaxuky & & & & & & & $\mathrm{X}$ & & & & & & $\mathrm{X}$ & $\mathrm{x}$ & $\mathrm{X}$ & & & \\
\hline \multirow[t]{2}{*}{ terçado } & sarakã & & & & & $\mathrm{x}$ & & $\mathrm{X}$ & & & & & $\mathrm{x}$ & & & & & & \\
\hline & saasara & $\mathrm{X}$ & & $\mathrm{X}$ & $\mathrm{x}$ & & $\mathrm{x}$ & & $\mathrm{x}$ & $\mathrm{X}$ & $\mathrm{X}$ & $\mathrm{x}$ & & $\mathrm{X}$ & $\mathrm{x}$ & $\mathrm{X}$ & $\mathrm{X}$ & $\mathrm{X}$ & $\mathrm{x}$ \\
\hline
\end{tabular}

Quadro 08: Amostra parcial de casos de variação geográfica (Fonte: LIMA-PADOVANI (2016, p. 97)).

A partir de dados como esses, listados no Quadro 08, observamos que os conceitos em Apurinã têm duas ou três formas variantes distribuídas entre as comunidades. Apesar dessas variantes serem usadas ativamente em algumas comunidades e não em outras, seguindo uma distribuição geográfica, na maioria das vezes, em uma mesma comunidade, alguns falantes reconhecem mais de uma dessas formas para designar um mesmo referente. Isso ocorre devido às constantes migrações entre as comunidades e contatos mais recentes entre seus membros, estimulados, por exemplo, pela necessidade frequente de homens irem buscar esposas em outras comunidades, cumprindo as regras tradicionais de casamento ${ }^{9}$.

Por sua vez, a variação geracional está relacionada a questões ligadas à idade ou geração a que pertence o falante. Em Apurinã há formas que são utilizadas apenas pelos falantes mais idosos e que somente alguns indivíduos da geração mais nova reconhecem, mas não usam. Esse fator contribui para que tenhamos formas coexistindo na língua no mesmo espaço geográfico.

\footnotetext{
${ }^{9}$ Os Apurinã possuem uma organização social norteada por regras tradicionais de casamento que dividem o povo em duas metades: Xiwapurynyry e os Meetymanety. As regras determinam que os casamentos devem ser realizados entre membros pertencentes a grupos diferentes.
} 
O conhecimento da idade dos falantes observados é indispensável para que se possa comparar as divergências entre o falar dos jovens em detrimento do falar dos mais idosos. Entretanto, em Apurinã a grande maioria dos falantes fluentes da língua encontra-se em uma faixa etária superior aos 30 anos. O que torna extremamente difícil obter dados com pessoas de uma faixa etária mais jovem. Em função disso, o fator geracional, considerado como uma das variáveis não linguísticas que estão associadas ao uso de algumas variantes linguísticas, em relação a outras, foi avaliado apenas parcialmente, já que, até o momento, não foi possível obter dados com falantes de uma faixa etária inferior a 30 anos. No Quadro 06 listamos alguns exemplos de variação geracional:

\begin{tabular}{|c|c|c|}
\hline \multirow[t]{2}{*}{ Nome em Português } & \multicolumn{2}{|c|}{ Nome em Apurinã } \\
\hline & Forma usada pelos jovens & Forma usada pelos idosos \\
\hline 1. cachorro do mato & awãtenyky & kapasãnyky \\
\hline 2. jacu & tũti & thãpukyry \\
\hline 3. jiboia & katatypi & kamatsupaãta \\
\hline \multirow[t]{2}{*}{ 4. sucurijú } & \multirow[t]{2}{*}{ kapinhuty } & kiãty \\
\hline & & wanhamary \\
\hline 5. nambu preto & takataka & surẽty \\
\hline 6. pé de tucumã & ikawaryna & xũkuruna \\
\hline 7. macaco de cheiro & xariwa & ipỹte \\
\hline 8. paca & kaiaty & txipama \\
\hline 9. carará & tarara & (k)ixiãryry \\
\hline 10. roçado $^{10}$ & tukury & kikiu \\
\hline
\end{tabular}

Quadro 09: Casos de variação geracional (Fonte: LIMA-PADOVANI (2006, p. 100).

A variação geracional está geralmente relacionada às mudanças linguísticas que acompanham cada geração. Quando duas ou mais palavras competem, uma delas pode entrar em desuso tornando-se um arcaísmo na língua ou adquirindo um novo significado,

10 Alguns colaboradores distinguem semanticamente kikiu de tukury. Para eles a forma kikiu designa 'roçado' enquanto tukury corresponde ao conceito 'derrubada' (processo que consiste na preparação do terreno para o cultivo). Tal fato sugere que a forma tukury ao longo do tempo passou também a designar 'roçado', caracterizandose como uma mudança em curso do significado do termo tukury. No que corresponde à forma kikiu, como podemos constatar no Quadro 06, ela está restrita à fala dos mais velhos nas comunidades em que ocorre (LIMAPADOVANI, 2016, p. 100). 
frequentemente relacionado semântica ou pragmaticamente ao significado original (ver LIMAPADOVANI, 2016).

Em Apurinã identificamos também a variação geográfico-geracional, que se caracteriza pela ocorrência de uma variação geracional em diferentes comunidades geográficas. Nesta última, uma variante que pode ter uma distribuição geográfica restrita também aparecer restrita a uma faixa etária específica. Como exemplo desse tipo de variação, podemos considerar o caso da palavra para 'macaco-de-cheiro', que é reconhecida por três formas: amãtxuary, ipỹte e xariwa. A forma amãtxuary está restrita a algumas comunidades, caracterizando aí uma variação geográfica; porém, mesmo nessas comunidades, essa forma encontra-se restrita a uma faixa etária específica, pois é uma forma antiga, usada somente pelos mais idosos, o que caracterizaria também nessas comunidades a variação geracional. No Quadro 10 listamos alguns desses exemplos:

\begin{tabular}{|l|l|l|}
\hline \multirow{2}{*}{ Nome em Português } & \multicolumn{2}{c|}{ Nome em Apurinã } \\
\cline { 2 - 3 } & $\begin{array}{l}\text { Forma usada pelos mais } \\
\text { jovens }\end{array}$ & Forma usada pelos mais velhos \\
\hline piquiá & samary & akanhi \\
\hline biorana & patxi & wakaru \\
\hline queixada & irary & ithumaneru \\
\hline timbó-açú & kunãa & itape \\
\hline macaco taboca & xariwa & amãtxuary; ipỹte \\
\hline
\end{tabular}

Quadro 10: Casos de variação geográfico-geracional (Fonte: Adaptado de LIMA-PADOVANI (2016)).

Lima-Padovani (2016) identificou, ainda, em Apurinã o fenômeno de "duplo vocabulário" 11 que consiste em itens lexicais que apresentam uma aparente sinonímia em que mais de uma palavra é usada para se referir ao mesmo elemento conceitual em um domínio específico do léxico. As propriedades semânticas de algumas unidades lexicais derivam de seu duplo estatuto de denominação; isto é, da possibilidade de designar um conceito a partir de dois parâmetros distintos, pois o sentido um termo não deriva apenas de propriedades semânticas dos seus referentes, mas também das determinações que lhe são conferidas pelo uso ou pela sua relação com outros termos do sistema lexical.

Um exemplo disso seria a forma como os Apurinã nomeiam o conceito 'piau quati'. O mesmo conceito pode ser chamado de puwana ou de kapixixima (kapixi 'quati' + xima nome

\footnotetext{
${ }^{11}$ Para mais informações acerca do fenômeno de "Duplo Vocabulário" atestado na língua Apurinã vê Lima-Padovani (2016).
} 
classificatório para peixes). A distinção entre os elementos desse par de nomes é que enquanto o primeiro é mais comumente usado no dia a dia, o segundo aparece em contextos mais restritos. Em geral, tal fenômeno ocorre quando os falantes desejam dar ênfase a uma propriedade específica do elemento em questão. No caso específico deste exemplo, os consultores Apurinã relataram que esse tipo de peixe tem listras ao longo do corpo que se parecem com as listras do rabo do 'quati'. Essa forma costuma ser empregada quando os falantes desejam enfatizar essa propriedade do 'piau quati'. Observe também a ocorrência de padrões metáforicos operando na construção dessa unidade lexical.

Diante de exemplos como o exposto acima, notamos que o "duplo vocabulário" apresenta uma coerência semântica construída a partir das relações metafóricas, metonímicas e pragmático-funcionais que motivam nomear dois referentes distintos com a mesma forma. Tal coerência sugere haver uma rede semântica interligando referentes distintos no léxico Apurinã, revelando assim traços da visão de mundo dos falantes. Essas relações evidenciam o quanto a interação é significativa no processo de formação das variantes lexicais. O "duplo vocabulário" é mais que um sistema de variação lexical, é um sistema tradicional de relações conceituais extremamente dinâmico em que alguns elementos dessa classificação são referidos e até classificados de formas aparentemente distintas, porém em pleno acordo com o modelo cognitivo e/ou cultural em que ele se encaixa dentro da sociedade, cultura, história e cosmovisão Apurinã.

\section{Conclusão}

Neste trabalho, buscamos descrever e analisar os distintos tipos de variação em três domínios da língua Apurinã, fonologia, morfologia e léxico, a fim de demonstrar a importância da diversidade da variação linguística presente no complexo dialetal Apurinã.

Apresentamos um panorama dos casos mais robustos de variação fonológica que marcam variedades distintas na língua Apurinã. Descrevemos alguns processos fonológicos envolvidos na formação das variantes desta língua. Identificamos que quando foi possível determinar a direção das mudanças vocálicas, elas podem ser descritas como instâncias de alteamento vocálico ou síncope; que nos casos envolvendo variação consonantal há o apagamento da glotal fricativa ou a variação entre consoantes líquidas, além da alternância do traço nasal entre sílabas adjacentes. Vimos também que os casos de variação morfológica envolvem, na sua maior parte, nomes classificatórios; marca de gênero; o paradigma pronominal de terceira pessoa singular e plural; e a redução da estrutura interna de algumas 
unidades lexicais. No que concerne à variação lexical, mostramos que há três subtipos de variação: a variação geográfica; a variação geracional e a variação geográfica-geracional.

Além disso, ainda em relação à variação lexical, vimos que o "duplo vocabulário" se constitui do uso extensivo de duas ou mais formas para designar um mesmo referente em um domínio específico do léxico. Com base na observação dos dados, concluímos que o "duplo vocabulário" codifica diferentemente alguns conceitos do seu universo e oferece recursos distintos para que o falante possa tratar esses conceitos. Algumas unidades lexicais do Apurinã se realizam em um discurso particular, resultado de uma construção sociocultural e uma escolha feita pelo falante, de acordo com a necessidade da situação comunicativa. Ou seja, a escolha que os falantes fazem de um item ou outro é influenciada pelo contexto.

Em linhas gerais, a configuação multidialetal em Apurinã está diretamente associada a distribuição geográfica das comunidades, que por sua vez está ligada ao constante movimento migratório e consequente contato e interação entre indivíduos de diferentes comunidades. Finalmente, nosso trabalho ilustra uma das dificuldades de se fazer pesquisa sociolinguística com línguas indígenas ameaçadas, em que o fato de a maioria dos falantes dessas línguas ser idosa impede a tradicional estratificação dos dados nos moldes da sociolinguística variacionista.

\section{Referências}

AIKHENVALD, A. Y. (2005). Arawak. In P. Strazny (ed.), Encyclopedia of Linguistics (pp. 81-84). New York: Fitzroy Dearborn.

BARRETO, Érica Lúcia. Variação em Apurinã: Aspectos Linguísticos e Fatores Condicionantes. Tese de mestrado. Universidade Federal do Pará. Belém: 2007.

BRANDÃO, Ana Paula B. Dicionário da Língua Apurinã. Trabalho de Conclusão de Curso (Licenciatura em Língua Portuguesa). Departamento de Letras e Literaturas Vernáculas, Universidade Federal do Pará. Belém: 2006.

CAMPBELL, Lyle. Historical Linguistics: an introduction. Cambridge: The MIT Press, 1999.

FACUNDES, Sidney da S. The Apurinã (Arawak) Language of Brazil. SUNY-Buffalo: Tese de Doutorado. 2000.

FACUNDES, Sidney da S; BRANDÃO, Ana Paula Barros. Hipóteses sobre o Desenvolvimento de Nasalidade Espontânea em uma Língua Aruák. Estudos Linguísticos. (São Paulo, 1978), Campinas, v. 34, n.XXXIV, p. xxx, 2005.

FACUNDES, Sidney da S.; NEVES, A. S. M.; LIMA-PADOVANI, Bruna Fernanda; Estudos 
Comparativos Sobre Apurinã Baseados em Documentos Antigos. Boletim do Museu Paraense Emílio Goeldi. Ciências Humanas, v 10, p. 143-158. 2015.

KOCH-GRÜNBERG, Theodor. Ein Beitrag zur Sprache der Ipuriná-Indianer. Journal de la Société des Americanistes. n. 11, P. 57-96, 1919.

KIBRIK; A.E. The Methodology of Field Investigations in Linguistics. The Hague: Mouton, 1977.

LABOV, William. Sociolinguistics Patterns. Philadelphia: University of Pennsylvania, 1972.

LIMA, Bruna Fernanda S. Variação, Mudanças e o "Duplo Vocabulário” na Língua Apurinã (Aruák). Belém, 2013. Trabalho de Conclusão de Curso (Licenciatura em Língua Portuguesa). Instituto de Letras e Comunicação, Universidade Federal do Pará, Belém.

LIMA-PADOVANI, Bruna Fernanda S. Levantamento Sociolinguístico do Léxico da Língua Apurinã e sua Contribuição para o Conhecimento da Cultura e História Apurinã (Aruák). Dissertação de Mestrado. Universidade Federal do Pará, Belém. 2016.

NIMUENDAJÚ, Curt. Vocabulários Makuší, Wapičána, Ipurinã' e Kapišanã'. Journal de la Société des Américanistes. Vol. 44. P. 179-197, 1955a.

POLAK, J.E.R, revisor. A Grammar and a Vocabulary of the Ipurinã Language. Vocabulary Publication Fund. N.1. London, 1894.

RAMIREZ, Henri. 2001. Línguas Arawak da Amazônia Setentrional. Manaus: Universidade do Amazonas.

RODRIGUES, Rayssa. A Língua Apurinã e os Efeitos de Contato com outras Línguas: A Fonologia. Relatório BIBIC/UFPA-AF ago/2014-jul/2015.

VAUX, Bert; COOPER, Justin. Introduction to Linguistic Field Methods. Lincom Europa, 1999.

Recebido em 30 de outubro de 2018. Aceito para publicação em 27 de dezembro de 2018. 